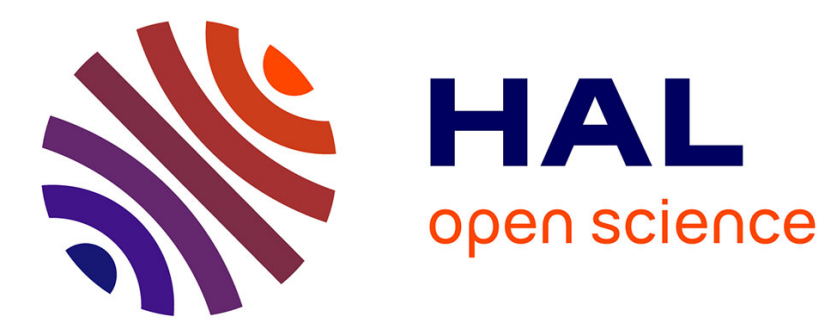

\title{
Épicondylalgies latérales dans une cohorte de salariés ligériens : évolution et déterminants
}

\author{
Alexis Descatha, Eléonore Herquelot, Zakia Mediouni, Audrey Petit, \\ Catherine Ha, Annette Leclerc, Yves Roquelaure
}

\section{To cite this version:}

Alexis Descatha, Eléonore Herquelot, Zakia Mediouni, Audrey Petit, Catherine Ha, et al.. Épicondylalgies latérales dans une cohorte de salariés ligériens : évolution et déterminants. Revue du Rhumatisme, 2014, 81 (4), pp.328-32. 10.1016/j.rhum.2013.10.006 . hal-03391142

HAL Id: hal-03391142

https://univ-angers.hal.science/hal-03391142

Submitted on 21 Oct 2021

HAL is a multi-disciplinary open access archive for the deposit and dissemination of scientific research documents, whether they are published or not. The documents may come from teaching and research institutions in France or abroad, or from public or private research centers.
L'archive ouverte pluridisciplinaire HAL, est destinée au dépôt et à la diffusion de documents scientifiques de niveau recherche, publiés ou non, émanant des établissements d'enseignement et de recherche français ou étrangers, des laboratoires publics ou privés. 
Article original

\title{
Épicondylalgies latérales dans une cohorte de salariés ligériens : évolution et déterminants
}

\author{
Alexis Descatha ${ }^{\mathrm{a}, \mathrm{b}, \mathrm{c}, *}$, Eleonore Herquelot ${ }^{\mathrm{a}, \mathrm{b}}$, Zakia Mediouni $^{\mathrm{a}, \mathrm{b}, \mathrm{c}}$, Audrey Petit $^{\mathrm{d}}$, \\ ${\text { Catherine } \mathrm{Ha}^{\mathrm{e}} \text {, Annette Leclerc }}^{\mathrm{a}, \mathrm{b}}$, Yves Roquelaure ${ }^{\mathrm{a}, \mathrm{b}}$ \\ a Université Versailles Saint-Quentin, 78035 Versailles, France \\ ' Centre de recherche en épidémiologie et santé des populations (CESP), Inserm, U1018, plate-forme de recherche "cohortes en population ", \\ 94807 Villejuif cedex, France \\ ' Unité de pathologie professionnelle/SAMU92, hôpitaux universitaires de Paris Île-de-France Ouest, site de Poincaré, AP-HP, 92380 Garches, France \\ d Laboratoire d'ergonomie et d'épidémiologie en santé au travail (LEEST), LUNAM université, université d'Angers, 49100 Angers, France \\ e Département santé travail, Institut de veille sanitaire (InVS), 94410 Saint-Maurice, France
}

\section{N F O A A R T I C L E}

\section{Historique de l'article :}

Accepté le 21 octobre 2013

Disponible sur Internet le 8 décembre 2013

\section{Mots clés :}

Douleur du coude

Épicondylalgie latérale

Épicondylite

Déclaration de maladie professionnelle

Facteurs professionnels

Maladie professionnelle

\begin{abstract}
R É S U M É
Objectifs. - Il n'existe que peu d'éléments concernant le pronostic à long terme des épicondylites latérales en population salariée en général. L'objectif a été d'écrire l'évolution et les déterminants des douleurs du coude et des épicondylalgies latérales vraisemblables dans une cohorte de salariés représentative de la population française au travail.

Méthode. - Des salariés ligériens présentant entre 2002 et 2004 une épicondylalgie latérale vraisemblable ont été suivis pour connaître l'évolution de leurs symptômes quatre ans après (2007 et 2009). Les déterminants possibles de la pathologie ont été étudiés.

Résultats. - Sur les 1611 salariés suivis, 100 sujets présentaient une épicondylalgie latérale vraisemblable en 2002-4. Plus d'un tiers des salariés inclus (39,0\%) présentaient une douleur au coude ou une épicondylalgie lors du second examen. Les travaux sollicitant le coude sont significativement associés à la persistance des épicondylalgies latérales vraisemblables en uni- et multivariée (odds ratio ajusté 5,59 $[1,47 ; 21,30])$.

Conclusion. - Les épicondylalgies latérales en milieu professionnel évoluent favorablement dans deux tiers des cas environ. Cette évolution dépend du diagnostic et de la prise en compte des facteurs professionnels dans la prise en charge. La déclaration de maladie professionnelle n'apparaît pas comme un déterminant indépendant significatif de mauvais pronostic même si le praticien doit évaluer les avantages et les inconvénients d'une telle démarche et en informer le patient.
\end{abstract}

@ 2013 Société Française de Rhumatologie. Publié par Elsevier Masson SAS. Tous droits réservés.

\section{Introduction}

Les épicondylalgies latérales sont un motif fréquent de consultation en population au travail et en population générale [1,2]. En France, elles sont une des premières causes de maladie professionnelle. Leur proportion est relativement stable alors que celle des affections péri-articulaires indemnisées en maladie professionnelle a augmenté depuis une dizaine d'années, (entre 18 et $20 \%$ pour le coude sur les cinq dernières années) [3]. Plusieurs études se sont intéressées à l'incidence et à la prévalence des pathologies de coude pour des secteurs d'activité professionnelle spécifiques. Dans une

\footnotetext{
* Auteur correspondant. Inserm U1018, UVSQ, unité de pathologie professionnelle U1018, CHU Poincaré, 104, boulevard Poincaré, 92380 Garches, France.

Adresse e-mail : alexis.descatha@uvsq.fr (A. Descatha).
}

cohorte agro-alimentaire, Kurppa et al. retrouvent un taux annuel d'incidence d'environ $10 \%$ ( 10 cas pour 100 personnes/année) chez les exposés et de $1 \%$ chez les non-exposés [4]. Pour des expositions manufacturières, les prévalences sont de l'ordre de 2 à $5 \%$ [5]. Shiri et al. retrouvent en population générale une prévalence de $3 \%$ pour l'épicondylite latérale et de $2 \%$ pour l'épicondylite médiale dans un échantillon de sujets de plus de 30 ans issu d'une enquête finlandaise en population générale [6], de même que Fan et al. [7]. Concernant les facteurs liés à l'évolution de la pathologie, des études menées auprès de patients après traitement médical ont conclu à une évolution favorable sans détailler les liens avec le travail $[1,8]$.

En dehors des résultats retrouvés chez des populations de travailleurs très exposés ou dans des séries cliniques, il existe peu d'éléments concernant le pronostic à long terme des épicondylites latérales en population salariée en général. 
L'objectif de cette étude est de décrire l'évolution des épicondylalgie latérales vraisemblables et d'étudier les déterminants de cette évolution dans une large cohorte de salariés française, représentative de la population française au travail.

\section{Méthode}

Depuis 2002, l'Institut de veille sanitaire (InVS) a mis en œuvre un programme de surveillance épidémiologique des troubles musculosquelettiques (TMS) dans la région des Pays-de-la-Loire et a permis d'obtenir un échantillon de salariés français représentatif de la population française au travail [9]. En effet, grâce à la participation de 83 médecins du travail, un échantillon de 3710 salariés âgés de 20 à 59 ans, dont $58 \%$ hommes, a été constitué par tirage au sort, entre 2002 et 2004 [10]. Près de $13 \%$ des salariés présentaient, le jour de la visite médicale du travail, au moins un des principaux TMS du membre dont une épicondylalgie latérale. Pour mémoire, les principaux TMS du membre supérieur sont la rupture de la coiffe des rotateurs, l'épicondylalgie latérale, le syndrome du nerf ulnaire, le syndrome du canal carpien, la tendinite de Quervain et la tendinite des extenseurs ou des fléchisseurs des doigts.

Un suivi longitudinal a été proposé en 2006 aux 3710 salariés inclus. La cohorte ainsi constituée est baptisée Cosali (Cohorte des salariés ligériens) [11,12]. En 2007, un questionnaire est adressé aux salariés par la poste. Il portait sur l'évaluation des symptômes musculosquelettiques. Un nouvel examen clinique a été réalisé par les médecins du travail entre 2007 et 2009.

L'échantillon de la présente étude a porté sur les patients présentant une épicondylalgie durant la phase d'inclusion entre 2002 et 2004. Il s'agissait de patients ayant rapporté une douleur persistante plus de 4 jours durant les 7 derniers jours ou une semaine au cours des 12 derniers mois et qui ont précisé, lors de l'examen clinique, une douleur intermittente dans la zone de l'épicondyle latéral. Ils ont été classés comme ayant une épicondylalgie latérale vraisemblable, suivant les recommandations internationales sur le sujet en milieu professionnel [13-15].

Le critère de jugement porte sur la notion d'épicondylalgie latérale vraisemblable lors du suivi entre 2007 et 2009 (répondant à la même définition qu'en 2002-2004). La présence d'autre type de douleur de coude a été également identifiée sur le même examen. Les sujets ont donc été classés lors du suivi en :

- pas de douleur de coude (référence);

- douleur de coude ne correspondant pas à une épicondylalgie latérale vraisemblable;

- épicondylalgie latérale vraisemblable.

Les variables étudiées sont issues de questionnaires de la cohorte (disponibles sur Internet [16,17]).

Les déterminants étudiés provenant de la phase d'inclusion entre 2002 et 2004 ont été les facteurs sociodémographiques, comme l'âge, le sexe, les facteurs professionnels et la position sociale.

La modification du poste de travail, le traitement, les pathologies associées et la déclaration de maladie professionnelle ont été recueillis pendant le suivi. On notera que les définitions des pathologies associées répondent aux critères internationaux de surveillance épidémiologique en milieu de travail. La notion de déclaration en maladie professionnelle repose sur le système actuel réglementaire français, avant les modifications en cours depuis 2011 du tableau correspondant [18].

Les méthodes statistiques ont utilisées des tests du chi ${ }^{2}$ pour les analyses univariées et des modèles de régression multinomiaux pour celles multivariées (SAS v9.2, SAS institute Inc, Cary, NC, ÉtatsUnis). Le seuil de significativité est à $5 \%$.

\section{Résultats}

Sur les 3710 individus initialement inclus entre 2002 et 2004 , 1611 (43,4\%) ont eu un suivi par un examen clinique entre 2007 et 2009 (sur les 2332 questionnaires reçus). Il n'existait pas de différence significative entre les personnes suivies et celles perdues de vue pour l'âge, le sexe, l'exposition professionnelle ou la présence de douleurs du coude [16,17]. Sur les 1611 salariés ayant bénéficié d'un suivi entre 2007 et 2009, 217 sujets présentaient des douleurs de coude entre 2002 et 2004 , et 100 présentaient une épicondylalgie latérale vraisemblable (échantillon étudié). La proportion de salariés avec épicondylalgie latérale vraisemblable à l'inclusion était similaire chez les sujets suivis que chez ceux perdus de vue (43,6\%, 100/216 dans ce dernier groupe).

À l'issue du suivi, $22,0 \%$ des cas présentaient toujours des épicondylalgies latérales probables et $17,0 \%$ des douleurs de coudes non épicondyliennes. Ainsi, seuls 61 cas ne présentaient pas de symptôme au coude lors du suivi.

La notion de traitement chirurgical dans la période et la présence de travaux sollicitant le coude semblaient associées significativement au fait de conserver des douleurs, notamment d'épicondylalgies latérales (Tableau 1). La présence de travaux sollicitant les coudes et la notion de traitement chirurgical dans la période étaient également significativement associées aux épicondylalgies latérales en analyse multivariée (Tableau 2). Une stratification sur l'aménagement du poste de travail ne modifiait pas l'association observée ni sa significativité.

La déclaration en maladie professionnelle a été peu fréquente et n'apparaît pas comme un déterminant significatif dans cette étude (Tableaux 1 et 2 ).

\section{Discussion}

Cette étude confirme l'impact des épicondylalgies latérales dans une cohorte française de travailleurs, puisqu'elles persistent dans plus d'un tiers des cas. La déclaration en maladie professionnelle a été peu réalisée et n'apparaît pas comme un déterminant indépendant significatif de mauvais pronostic contrairement aux facteurs d'exposition professionnelle.

La persistance importante des douleurs du coude et des épicondylalgies dans cette étude est compatible avec les résultats des études réalisées dans un contexte similaire. Dans une cohorte de salariés de l'industrie automobile, Werner et al. retrouvent une guérison des épicondylites dans les 13 mois dans $58 \%$ des cas [19]. Une étude longitudinale de salariés fortement exposés aux contraintes biomécaniques avait noté l'importance de la chronicisation des pathologies du membre supérieur: parmi 73 cas d'épicondylites latérales observées en 1993-94, 38 \% présentaient encore des signes cliniques d'épicondylites latérales en 1996-97 [20]. Le pronostic est similaire à celui retrouvé dans des études sur des populations consultantes pour un problème de santé soit en soins primaires ou secondaires [1,21], témoignant indirectement également d'un accès aux soins optimal y compris à celui du spécialiste dans cette région.

Les déterminants du pronostic retrouvés dans cette étude sont ceux retrouvés dans les autres TMS. Dans un essai sur la rééducation fonctionnelle de 266 patients atteints d'épicondylite latérale, Haahr et Andersen notent qu'un plus mauvais pronostic médical est observé chez les patients ayant un travail manuel [22]. Ce résultat est aussi retrouvé par Lewis et al. [23] et Waugh et al. en 2004 chez les femmes [8]. Une précédente étude réalisée sur la population source de l'échantillon a montré que le travail en force était le facteur pronostique principal de ces pathologies [24]. Une étude américaine concernant le syndrome du canal carpien retrouve un lien important entre la pathologie et l'exposition aux 
Tableau 1

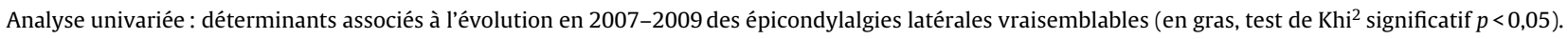

\begin{tabular}{|c|c|c|c|c|}
\hline & $\begin{array}{l}\text { Pas de douleur de coude } \\
\text { en } 2007-2009(n=61)\end{array}$ & $\begin{array}{l}\text { Douleurs de coude non } \\
\text { épicondyliennes en 2007-2009 } \\
(n=17)\end{array}$ & $\begin{array}{l}\text { Épicondylalgies latérales } \\
\text { vraisemblables en 2007-2009 } \\
(n=22)\end{array}$ & $\begin{array}{l}\text { Total suivi } \\
(n=100)\end{array}$ \\
\hline \multicolumn{5}{|l|}{ Sexe } \\
\hline Hommes & $35(57,38 \%)$ & $10(58,82 \%)$ & $15(68,18 \%)$ & $60(60,00 \%)$ \\
\hline Femmes & $26(42,62 \%)$ & $7(41,18 \%)$ & $7(31,82 \%)$ & $40(40,00 \%)$ \\
\hline \multicolumn{5}{|l|}{ Classes d'âge } \\
\hline$<30$ ans & $6(9,84 \%)$ & $1(5,88 \%)$ & $1(4,55 \%)$ & $8(8,00 \%)$ \\
\hline $30-44$ ans & $21(34,43 \%)$ & $8(47,06 \%)$ & $7(31,82 \%)$ & $36(36,00 \%)$ \\
\hline$>44$ ans & $34(55,74 \%)$ & $8(47,06 \%)$ & $14(63,64 \%)$ & $56(56,00 \%)$ \\
\hline \multicolumn{5}{|l|}{ Profession et catégorie socioprofessionnelle } \\
\hline Agriculteurs, artisans, commerçants & $20(32,79 \%)$ & $3(17,65 \%)$ & $6(27,27 \%)$ & $29(29,00 \%)$ \\
\hline Employés & $11(18,03 \%)$ & $6(35,29 \%)$ & $3(13,64 \%)$ & $20(20,00 \%)$ \\
\hline Ouvriers qualifiés & $16(26,23 \%)$ & $2(11,76 \%)$ & $8(36,36 \%)$ & $26(26,00 \%)$ \\
\hline Ouvriers non qualifiés & $14(22,95 \%)$ & $6(35,29 \%)$ & $5(22,73 \%)$ & $25(25,00 \%)$ \\
\hline \multicolumn{5}{|l|}{ Antécédents d'épicondylalgie latérale } \\
\hline Non & $35(57,38 \%)$ & $10(58,82 \%)$ & $10(45,45 \%)$ & $55(55,00 \%)$ \\
\hline Oui & $26(42,62 \%)$ & $7(41,18 \%)$ & $12(54,55 \%)$ & $45(45,00 \%)$ \\
\hline \multicolumn{5}{|c|}{ Autre pathologie du membre supérieur associée } \\
\hline Non & $40(65,57 \%)$ & $13(76,47 \%)$ & $13(59,09 \%)$ & $66(66,00 \%)$ \\
\hline Oui & $21(34,43 \%)$ & $4(23,53 \%)$ & $9(40,91 \%)$ & $34(34,00 \%)$ \\
\hline \multicolumn{5}{|l|}{ Droitier } \\
\hline Non & $8(13,11 \%)$ & $2(9,09 \%)$ & $1(5,88 \%)$ & $11(11,00 \%)$ \\
\hline Oui & $53(86,89 \%)$ & $20(90,91 \%)$ & $16(94,12 \%)$ & $89(89,00 \%)$ \\
\hline \multicolumn{5}{|l|}{ Déclaration en maladie professionnelle } \\
\hline Non & $54(88,52 \%)$ & $15(88,24 \%)$ & $17(77,27 \%)$ & $86(86,00 \%)$ \\
\hline Oui & $7(11,48 \%)$ & $2(11,76 \%)$ & $5(22,73 \%)$ & $14(14,00 \%)$ \\
\hline \multicolumn{5}{|l|}{ Travaux sollicitant le coude } \\
\hline Non & $35(57,38 \%)$ & $7(41,18 \%)$ & $5(22,73 \%)$ & $47(47,00 \%)$ \\
\hline Oui & $26(42,62 \%)$ & $10(58,82 \%)$ & $17(77,27 \%)$ & $53(53,00 \%)$ \\
\hline \multicolumn{5}{|l|}{ Traitement antalgique palier 3} \\
\hline Non & $59(96,72 \%)$ & $16(94,12 \%)$ & $22(100,0 \%)$ & $97(97,00 \%)$ \\
\hline Oui & $1(1,64 \%)$ & $1(5,88 \%)$ & $0(0,00 \%)$ & $2(2,00 \%)$ \\
\hline \multicolumn{5}{|c|}{ Antécédent de chirurgie du membre supérieur } \\
\hline Non & $54(88,52 \%)$ & $14(82,35 \%)$ & $15(68,18 \%)$ & $83(83,00 \%)$ \\
\hline Oui & $6(9,84 \%)$ & $3(17,65 \%)$ & $7(31,82 \%)$ & $16(16,00 \%)$ \\
\hline \multicolumn{5}{|l|}{ Antécédents d'infiltration } \\
\hline Non & $57(93,44 \%)$ & $16(94,12 \%)$ & $20(90,91 \%)$ & $93(93,00 \%)$ \\
\hline Oui & $3(4,92 \%)$ & $1(5,88 \%)$ & $2(9,09 \%)$ & $6(6,00 \%)$ \\
\hline
\end{tabular}

travaux en force même si le principal facteur pronostique identifié est la probabilité diagnostique, comme ici illustrée par la notion d'épicondylalgie avérée ou non [25].

Les traitements de la pathologie apparaissent comme un facteur de risque de l'évolution naturelle de la pathologie. Il n'est bien sûr pas possible de conclure au vu du type d'étude. Le traitement chirurgical est ici probablement le témoin d'une résistance au traitement médical et un indicateur indirect de mauvais pronostic plutôt qu'un facteur aggravant.

Il n'est pas retrouvé de lien entre déclaration en maladie professionnelle et pronostic des épicondylalgies. La notion d'indemnisation en maladie professionnelle est très différente de celle d'une maladie potentiellement liée au travail. D'une part, le tableau de maladie professionnelle est le fruit d'un

Tableau 2

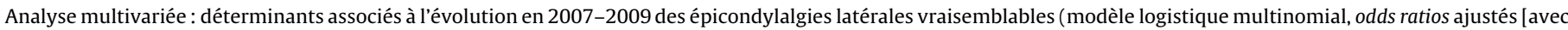
intervalle de confiance à $95 \%$ ]).

\begin{tabular}{|c|c|c|}
\hline & $\begin{array}{l}\text { Odds ratios ajustés des douleurs du coude non } \\
\text { épicondyliennes en 2007-2009 (versus pas de } \\
\text { douleur de coude) }\end{array}$ & $\begin{array}{l}\text { Odds ratios ajustés des épicondylalgies } \\
\text { latérales vraisemblables en 2007-2009 (versus } \\
\text { pas de douleur de coude) }\end{array}$ \\
\hline \multicolumn{3}{|l|}{ Sexe } \\
\hline Hommes & 1 & 1 \\
\hline Femmes & $1,16[0,36 ; 3,81]$ & $0,91[0,27 ; 3,09]$ \\
\hline \multicolumn{3}{|l|}{ Classes d'âge (en 2002-4) } \\
\hline$<30$ ans & 1 & 1 \\
\hline $30-44$ ans & $1,79[0,18 ; 18,01]$ & $1,14[0,09 ; 14,28]$ \\
\hline$>44$ ans & $1,08[0,10 ; 11,22]$ & $1,55[0,13 ; 18,26]$ \\
\hline \multicolumn{3}{|l|}{ Travaux sollicitant le coude } \\
\hline Non & 1 & 1 \\
\hline Oui & $1,91[0,58 ; 6,34]$ & $5,59[1,47 ; 21,30]$ \\
\hline \multicolumn{3}{|c|}{ Traitement entre $2002-4$ et 2007-10) } \\
\hline Pas de traitement & 1 & 1 \\
\hline Traitement médical & $1,57[0,42 ; 5,93]$ & $3,04[0,84 ; 11,03]$ \\
\hline Traitement chirurgical & $3,18[0,54 ; 18,75]$ & $9,64[1,87 ; 49,63]$ \\
\hline \multicolumn{3}{|c|}{ Déclaration en maladie professionnelle (entre 2002-4 et 2007-10) } \\
\hline Non & 1 & 1 \\
\hline Oui & $0,62[0,09 ; 4,15]$ & $1,13[0,24 ; 5,26]$ \\
\hline
\end{tabular}


consensus médical, mais surtout économique et social, lors de sa création ou de sa révision et donc n'est pas exhaustif. D'autre part, il existe de nombreux cas où il y a plus d'inconvénients médicaux et sociaux à la déclaration en maladie professionnelle, raison pour laquelle moins d'un quart a été déclaré en maladie professionnelle. De précédents travaux sur le canal carpien [26] ont montré que la déclaration en elle-même n'avait probablement pas, si elle était conseillée correctement par le praticien, d'effet direct sur le pronostic lorsqu'il existe une prise en compte des facteurs socioprofessionnels. Cet effet existait à court terme uniquement dans une étude rétrospective chirurgicale [21].

En dehors de l'effectif réduit, les limites de l'étude sont principalement sur des effets de sélection liés aux perdus de vue. Les perdus de vue ne semblent pas être différents des patients suivis pour les caractéristiques initiales. Des effets de déclaration sont à évoquer puisque les expositions sont auto-déclarées de même que la pathologie (douleur à l'interrogatoire). Cette proportion est attendue car elle témoigne d'une réalité en France liée à la situation sociale et économique des entreprises, mais également la situation démographique des médecins du travail. Cette étude est tout à fait représentative de cette situation et une analyse récente autour d'incidence des pathologies avec imputations multiples confirme l'absence probable d'effet de sélection [27]. Même s'il est toujours possible de discuter la valeur diagnostique réelle des critères diagnostiques utilisés (douleur au coude, épicondylalgie latérale, autre TMS), la validité des critères diagnostiques comme celle des critères d'évaluation de l'exposition, ont été s avant d'être proposées au niveau européen [13]. Le design longitudinal permet d'éliminer d'éventuels biais de classement différentiel puisque l'exposition a été associée au devenir clinique plusieurs années après. L'effectif limité et le manque d'information sur les différentes thérapeutiques, comme le type d'aménagement de poste sont aussi des limites importantes de l'étude.

En conclusion, les épicondylalgies latérales en milieu professionnel évoluent favorablement dans 60 à $70 \%$ des cas en fonction de la prise en compte des facteurs professionnels lors de la prise en charge médicale. La déclaration en maladie professionnelle n'apparaît pas comme un déterminant indépendant significatif de mauvais pronostic, même s'il est important d'évaluer les avantages et inconvénients d'une telle déclaration et d'en informer le patient. D’autres études sont nécessaires pour connaître l'impact des traitements médicaux et chirurgicaux chez ces populations particulières de sujet au travail, comme la qualité de vie et les facteurs psychologiques.

\section{Déclaration d'intérêts}

Les auteurs déclarent ne pas avoir de conflits d'intérêts en relation avec cet article.

\section{Remerciements}

Les auteurs expriment leurs remerciements aux médecins du travail des Pays-de-la-Loire qui ont participé à cette étude: Docteurs Abonnat, Banon, Bardet, Becquemie, Bertin, Bertrand, Bidron, Biton, Bizouarne, Boisse, Bonamy, Bonneau, BouguerDiquelou, Bourrut-Lacouture, Breton, Caillon, Cesbron, Chisacof, Chotard, Compain, Coquin-Georgeac, Cordes, Couet, Coutand, Danielou, Darcy, Davenas, De Lansalut, De Lescure, Diquelou, Dopsent, Dufrenne-Benetti, Dupas, Evano, Fache, Fontaine, Frampas-Chotard, Guillier, Guillimin, Harinte, Harrigan, Hervio, Hirigoyen, Jahan, Jube, Kalfon, Labraga, Laine-Colin, Laventure, Le Dizet, Lechevalier, Le Clerc, Ledenvic, Leroux, Leroy-Maguer,
Levrard, Levy, Logeay, Lucas, Mallet, Martin-Laurent, Mazoyer, Meritet, Michel, Migne-Cousseau, Moisan, Page, Patillot, Pinaud, Pineau, Pizzalla, Plessis, Plouhinec, Raffray, Robin-Riom, Roussel, Russu, Saboureault, Schlindwein, Soulard, Thomson, Treillard et Tripodi.

Ce projet est financé par l'Institut de veille sanitaire (9/25/20025 "Réseau expérimental de surveillance des troubles musculosquelettiques») et le projet Temis (Anses Agence française pour l'alimentation, de la santé environnementale et professionnelle, APR EST 2011 TEMIS).

\section{Références}

[1] Smidt N, Lewis MVAN, Windt DER, et al. Lateral epicondylitis in general practice: course and prognostic indicators of outcome. J Rheumatol 2006;33:2053-9.

[2] Herquelot E, Bodin J, Roquelaure Y, et al. Work-related risk factors for lateral epicondylitis and other cause of elbow pain in the working population. Am J Ind Med 2013;56:400-9.

[3] Descatha A, Roquelaure Y, Leclerc A. Épidemiologie des pathologies d'hypersollicitation du coude et du syndrome du nerf ulnaire au coude d'origine professionnelle; Coude Pathol Prof. Paris: Masson; 2008. p. $62-75$.

[4] Kurppa K, Viikari-Juntura E, Kuosma E, et al. Incidence of tenosynovitis or peritendinitis and epicondylitis in a meat-processing factory. Scand J Work Environ Health 1991;17:32-7.

[5] Hagberg M, Silverstein BA, Wells R, et al. Work-related musculoskeletal disorders (WMSDs). A reference book for prevention. Bristol: Taylor and Francis; 1995.

[6] Shiri R, Viikari-Juntura E, Varonen H, et al. Prevalence and determinants of lateral and medial epicondylitis: a population study. Am J Epidemiol 2006; 164:1065-74.

[7] Fan Z], Silverstein BA, Bao S, et al. Quantitative exposure-response relations between physical workload and prevalence of lateral epicondylitis in a working population. Am J Ind Med 2009;52:479-90.

[8] Waugh EJ, Jaglal SB, Davis AM, et al. Factors associated with prognosis of lateral epicondylitis after 8 weeks of physical therapy. Arch Phys Med Rehabil 2004;85:308-18.

[9] Roquelaure Y, Ha C, Leclerc A, et al. Epidemiologic surveillance of upperextremity musculoskeletal disorders in the working population. Arthritis Rheum 2006:55:765-78.

[10] Descatha A, Roquelaure Y, Ha C, et al. Surveillance épidémiologique des pathologies d'hypersollicitation du membre supérieur d'origine professionnelle. Arch Mal Prof Environ 2007;68:153-60.

[11] Bodin J, Ha C, Petit Le Manac'h A, et al. Risk factors for incidence of rotator cuff syndrome in a large working population. Scand J Work Environ Health 2012;38:436-46

[12] Bodin J, Ha C, Sérazin C, et al. Effects of individual and work-related factors on incidence of shoulder pain in a large working population. J Occup Health 2012;54:278-88.

[13] Sluiter BJ, Rest KM, Frings-Dresen MH. Criteria document for evaluating the work-relatedness of upper-extremity musculoskeletal disorders. Scand J Work Environ Health 2001;27:1-102.

[14] Aublet-Cuvelier A, Ha C, Roquelaure Y, et al. Protocole d'examen clinique pour le repérage des troubles musculosquelettiques du membre supérieur. Adaptation française du consensus européen SALTSA. Études Doc 2007;4254: $1-31$.

[15] Meyer JD, Chen Y, McDonald JC, et al. Surveillance for work-related hearing loss in the UK: OSSA and OPRA 1997-2000. Occup Med (Lond) 2002;52: 75-9.

[16] http://opac.invs.sante.fr/doc_num.php?explnum_id=3662

[17] http://ead.univ-angers.fr/ leest/spip.php?rubrique76

[18] Descatha A, Thomas T, Aubert F, et al. Tendinopathie calcifiante de l'épaule et maladie professionnelle : maladie calcifiante tendineuse ou tendinopathie avec calcification? Presse Med 2012;41:453-4.

[19] Werner RA, Franzblau A, Gell N, et al. Predictors of upper extremity discomfort: a longitudinal study of industrial and clerical workers. J Occup Rehabil 2005; 15:27-35.

[20] Descatha A, Roquelaure Y, Chastang JF, et al. Description of outcomes of upperextremity musculoskeletal disorders in workers highly exposed to repetitive work. J Hand Surg Am 2009;34:890-5.

[21] Bigorre N, Raimbeau G, Fouque P-A, et al. Lateral epicondylitis treatment by extensor carpi radialis fasciotomy and radial nerve decompression: is outcome influenced by the occupational disease compensation aspect? Orthop Traumatol Surg Res 2011;97:159-63.

[22] Haahr JP, Andersen JH. Prognostic factors in lateral epicondylitis: a randomized trial with one-year follow-up in 266 new cases treated with minimal occupational intervention or the usual approach in general practice. Rheumatology (Oxford) 2003:42:1216-25.

[23] Lewis M, Hay EM, Paterson SM, et al. Effects of manual work on recovery from lateral epicondylitis. Scand J Work Env Health 2002;28: 109-16. 
[24] Descatha A, Roquelaure Y, Chastang JF, et al. Work, a prognosis factor for upper extremity musculoskeletal disorders? Occup Environ Med 2009;66: $351-2$.

[25] Descatha A, Dale AM, Franzblau A, et al. Natural history and predictors of longterm pain and function among workers with hand symptoms. Arch Phys Med Rehabil 2013;94:1293-9.
[26] Parot-Schinkel E, Roquelaure Y, Ha C, et al. Factors affecting return to work after carpal tunnel syndrome surgery in a large French cohort. Arch Phys Med Rehabil 2011;92:1863-9

[27] Herquelot E, Guegen A, Roquelaure Y, et al. Work-related risk factors for incidence of lateral epicondylitis in a large working population. Scand J Work Env Heal 2013, 10.5271/sjweh.3380. 